\title{
Absolute Method to Certify Quantum Yields of Photon Upconversion via Triplet-Triplet
}

\section{Annihilation}

Nobuhiro Yanai, ${ }^{*}{ }^{\dagger},+$ Kengo Suzuki, ${ }^{*}{ }^{*}$ Taku Ogawa, ${ }^{\dagger}$ Yoichi Sasaki, ${ }^{\dagger}$ Naoyuki Harada, ${ }^{\dagger}$ and Nobuo Kimizuka ${ }^{* \dagger}$

†Department of Chemistry and Biochemistry, Graduate School of Engineering, Center for Molecular Systems (CMS), Kyushu University, 744 Moto-oka, Nishi-ku, Fukuoka 819-0395, Japan.

†PRESTO, JST, Honcho 4-1-8, Kawaguchi, Saitama 332-0012, Japan.

${ }^{\#}$ Systems Division, Hamamatsu Photonics K. K., 812 Joko-cho, Higashi-ku, Hamamatsu-city, 431-8196.

* To whom corresponding should be addressed. E-mail: yanai@mail.cstm.kyushu-u.ac.jp (N.Y.); kengo-s@sys.hpk.co.jp (K.S.); n-kimi@ mail.cstm.kyushu-u.ac.jp (N.K.) 


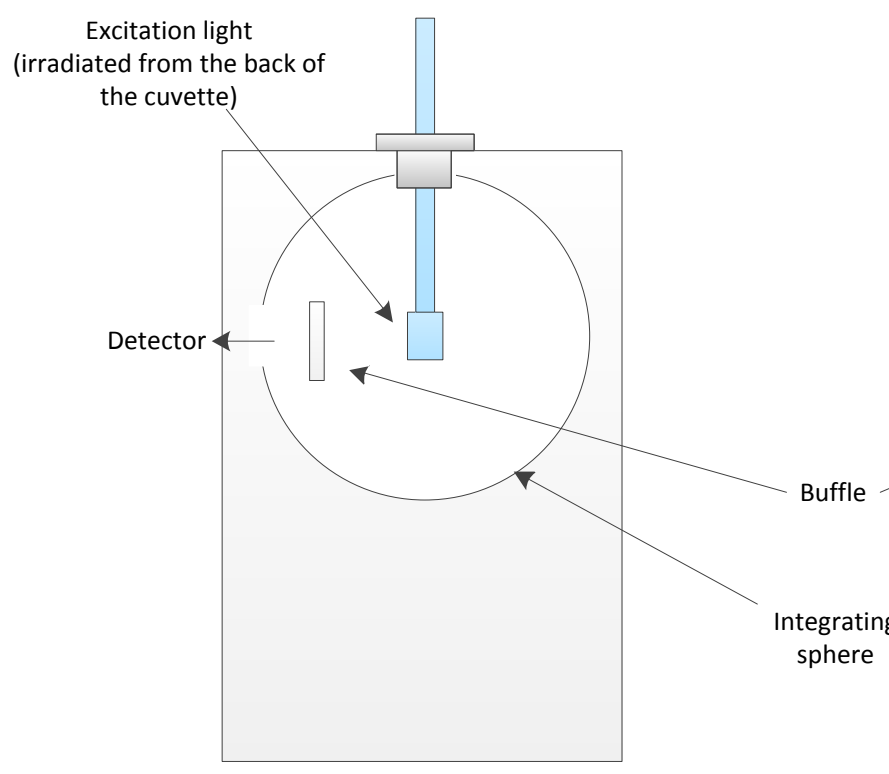

Side view

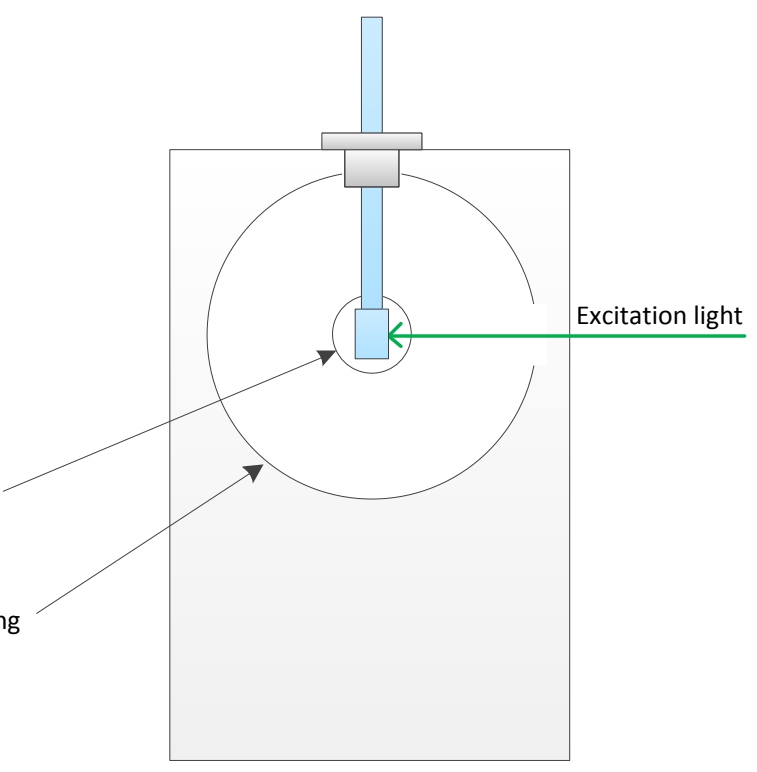

Front view

Figure S1. Schematic illustration of absolute quantum yield measurement setup.

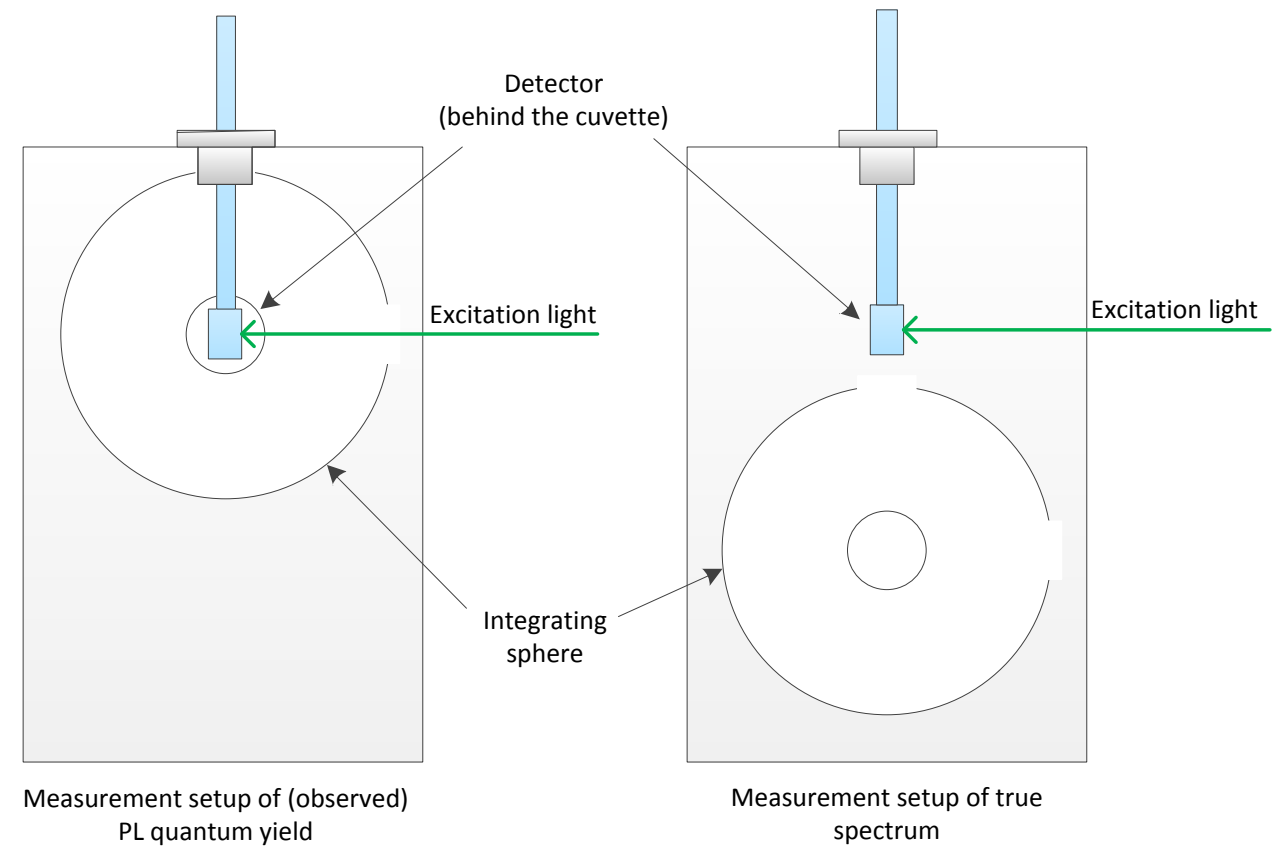

Figure S2. Schematic illustration of the absolute quantum yield measurement setup with mechanically removable integrating sphere for Experiment (A) (left) and Experiment (B) (right). 


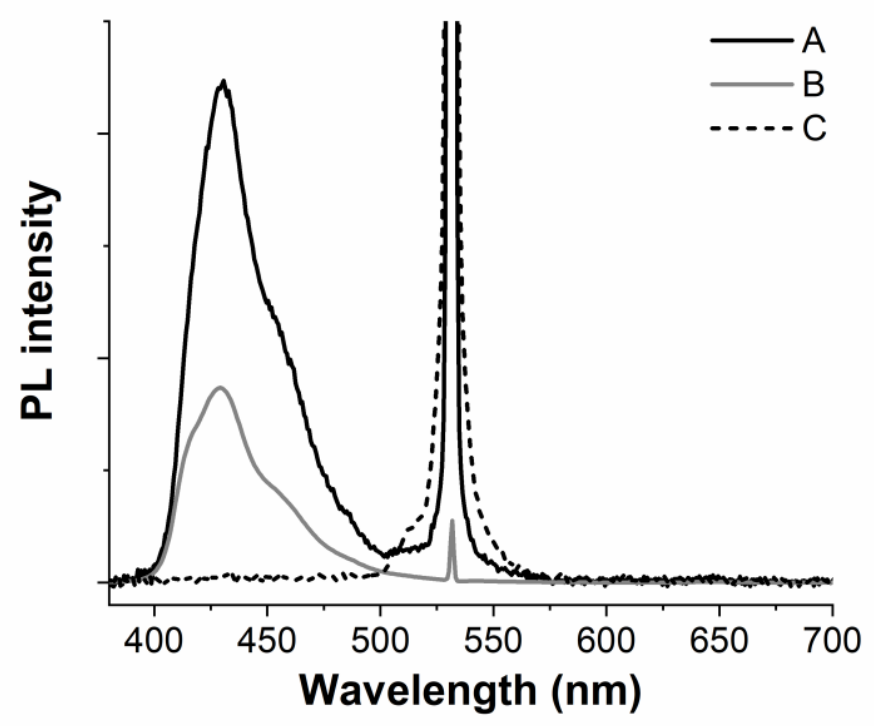

Figure S3. Photoluminescence (PL) spectra of PtOEP $(100 \mu \mathrm{M})$ and DPA $(10 \mathrm{mM})$ in deaerated THF in Experiment (A), (B) and (C) normalized to identical exposure time without a short-pass filter $\left(\lambda_{\mathrm{ex}}=532 \mathrm{~nm}, I_{\mathrm{ex}}=5.7 \mathrm{~mW} / \mathrm{cm}^{2}\right)$. The TTA-UC emission intensity in Experiment $(\mathrm{C})$ was much smaller than those in Experiment (A) and (B). Note that the TTA-UC emission intensity in Experiment (A) is larger than the one in Experiment (B), but this trend becomes opposite when the emission intensity is scaled based on the PtOEP phosphorescence (Figure 2c, 2d).

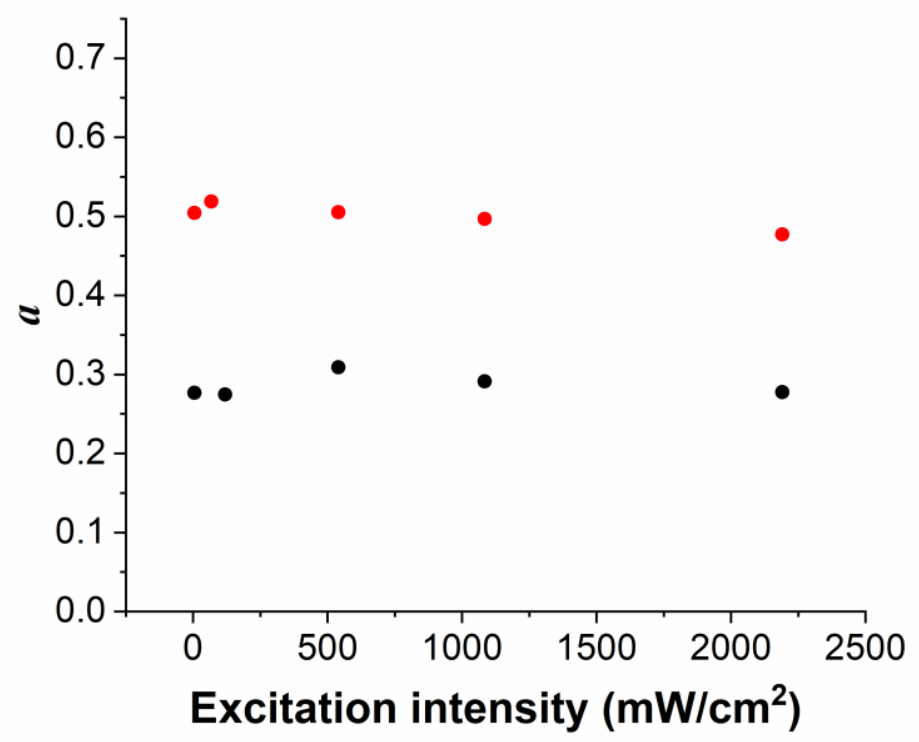

Figure S4. Reabsorption probability $a$ of PtOEP $(100 \mu \mathrm{M})$ and DPA $(10 \mathrm{mM})$ in deaerated THF with sample volume of $1 \times 4 \times 7 \mathrm{~mm}^{3}$ (black) and $1 \times 10 \times 35 \mathrm{~mm}^{3}$ (red) as a function of excitation intensity. 
(a)

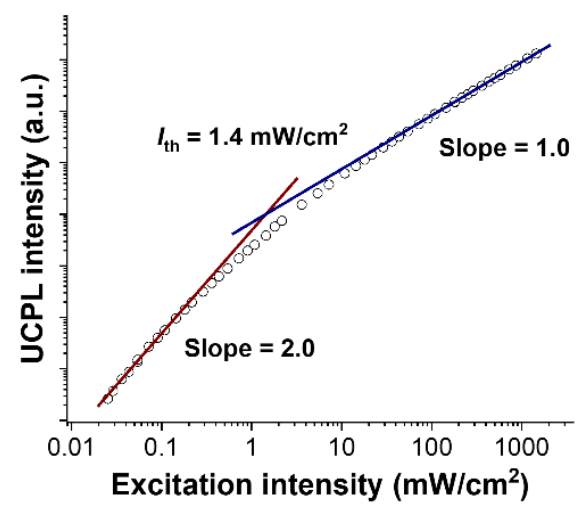

(b)

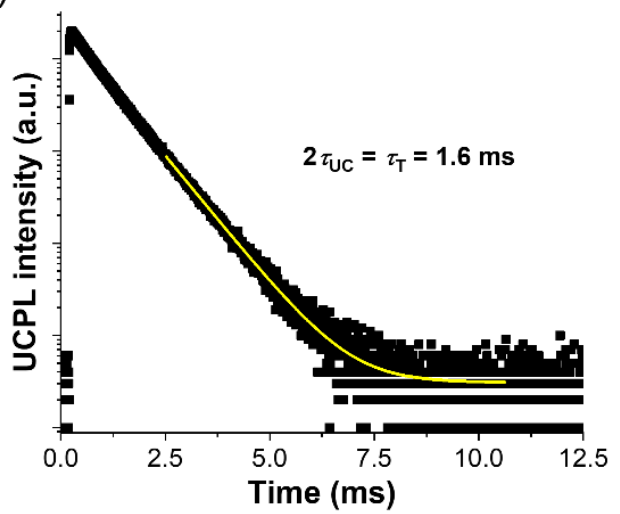

Figure S5. (a) TTA-UC emission intensity of a PtOEP-DPA THF solution ([PtOEP] $=100 \mu \mathrm{M}$, $[\mathrm{DPA}]=10 \mathrm{mM}$ ) as a function of excitation intensity. (b) TTA-UC emission decay of the PtOEP-DPA THF solution upon pulsed excitation at $531 \mathrm{~nm}\left(\lambda_{\mathrm{em}}=430 \mathrm{~nm}\right.$, [PtOEP] $=100 \mu \mathrm{M}$, $[\mathrm{DPA}]=10 \mathrm{mM})$. The fitting curve was obtained by the known relationship of $I_{\mathrm{UC}}(t) \propto$ $\exp \left(-t / \tau_{\mathrm{UC}}\right)=\exp \left(-2 t / \tau_{\mathrm{T}}\right)$, where $\tau_{\mathrm{UC}}$ and $\tau_{\mathrm{T}}$ are lifetimes of TTA-UC emission and emitter triplet, respectively.

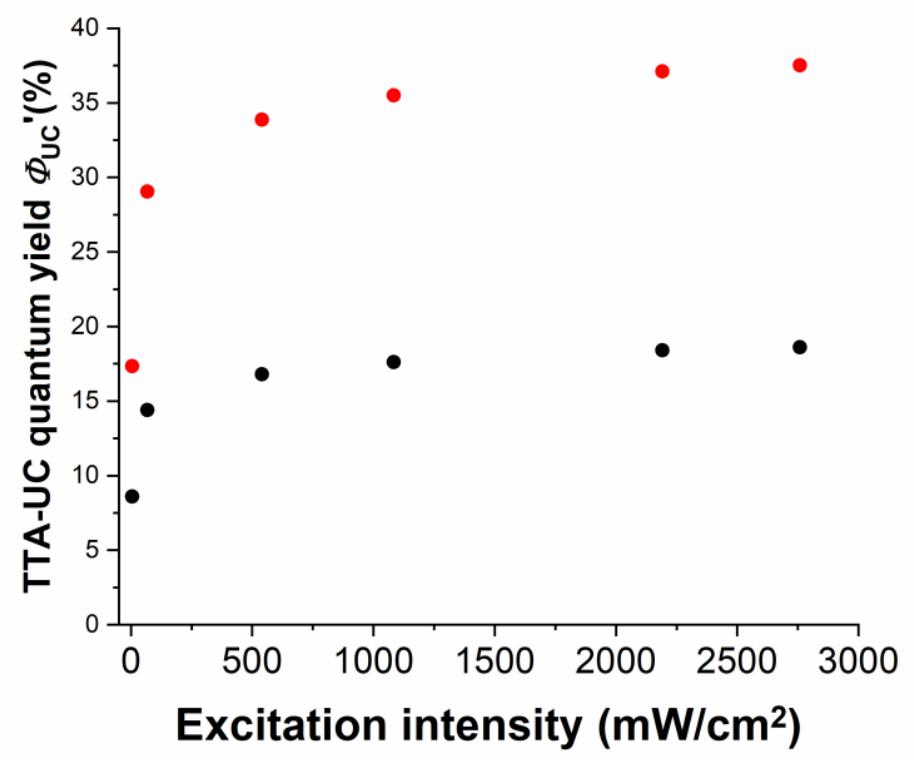

Figure S6. Absolute TTA-UC quantum yield $\Phi_{\mathrm{UC}}$ ' of PtOEP $(100 \mu \mathrm{M})$ and DPA $(10 \mathrm{mM})$ in deaerated THF without (black) and with (red) the reabsorption correction (sample volume $=$ 
$\left.1 \times 10 \times 35 \mathrm{~mm}^{3}\right)$.

(a)

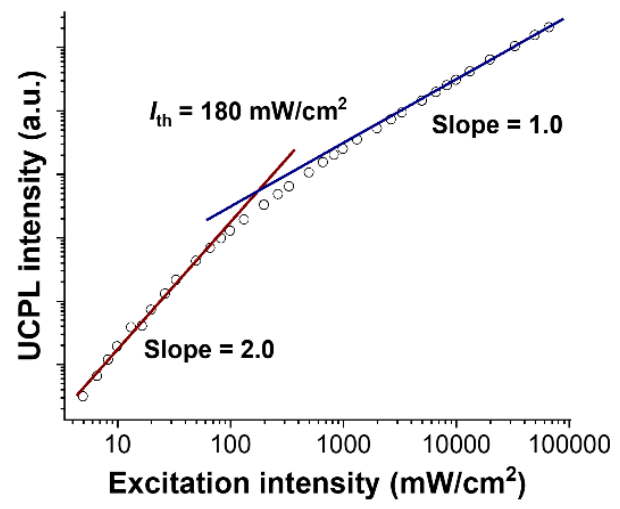

(b)

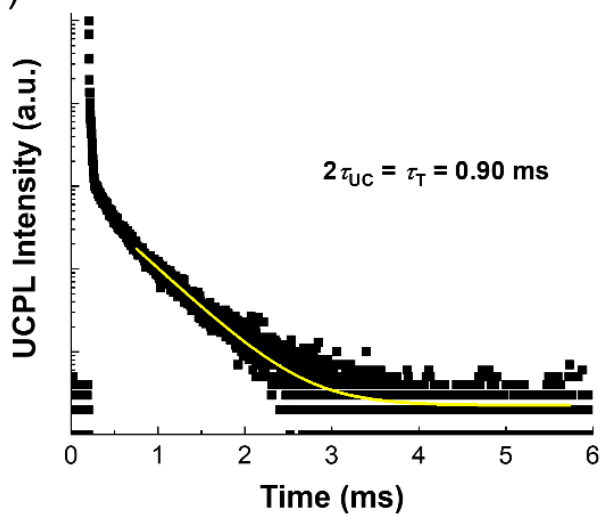

Figure S7. (a) TTA-UC emission intensity of a PtTPBP-TTBP THF solution ([PtTPBP] $=100$ $\mu \mathrm{M},[\mathrm{TTBP}]=10 \mathrm{mM}$ ) as a function of excitation intensity. (b) TTA-UC emission decay of the PtTPBP-TTBP THF solution upon pulsed excitation at $630 \mathrm{~nm}\left(\lambda_{\mathrm{em}}=485 \mathrm{~nm}\right.$, $[\mathrm{PtTPBP}]=100$ $\mu \mathrm{M},[\mathrm{TTBP}]=10 \mathrm{mM})$. The fitting curve was obtained by the known relationship of $I_{\mathrm{UC}}(t) \propto$ $\exp \left(-t / \tau_{\mathrm{UC}}\right)=\exp \left(-2 t / \tau_{\mathrm{T}}\right)$, where $\tau_{\mathrm{UC}}$ and $\tau_{\mathrm{T}}$ are lifetimes of TTA-UC emission and emitter triplet, respectively. 